\title{
ICNIRP NOTE: CRITICAL EVALUATION OF TWO RADIOFREQUENCY ELECTROMAGNETIC FIELD ANIMAL CARCINOGENICITY STUDIES PUBLISHED IN 2018
}

\author{
International Commission on Non-Ionizing Radiation Protection (ICNIRP)
}

\begin{abstract}
Final results are now available from two large animal studies that investigated whether long-term exposure to radiofrequency (RF) electromagnetic fields (EMFs) associated with mobile (or cell) phones or base stations is carcinogenic; these studies hale from the US National Toxicology Program (NTP) and the Ramazzini Institute in Italy, respectively. In both cases, the authors concluded that they had demonstrated that RF EMFs are carcinogenic in male rats but not in female rats or mice (NTP only). The International Commission on Non-Ionizing Radiation Protection (ICNIRP) has evaluated their methods and findings for potential information about the carcinogenicity of exposure to RF EMFs. We found that these studies had important strengths; for example, both followed good laboratory practice (GLP), both used much larger numbers of animals than previous research, and both exposed animals over the whole of their lives. We also noted some major weaknesses, including a lack of blinding, difficulties interpreting statistical analyses due to the association between longer lifespans and tumor occurrence in the exposed rats (NTP only), and failure to account for chance. ICNIRP concluded that these substantial limitations preclude conclusions being drawn concerning RF EMFs and carcinogenesis. Health Phys. 118(00):000-000; 2020
\end{abstract}

Key words: carcinogenesis; exposure, radiofrequency; radiation protection; International Commission on Non-Ionizing Radiation Protection (ICNIRP)

\section{INTRODUCTION}

RADIOFREQUENCY (RF) electromagnetic fields (EMFs) are utilized to enable a range of wireless technologies to function, such as FM radio, radar, wireless routers, and mobile (cell) phones and associated base stations. As a result, humans are regularly exposed to RF EMFs, making it important to understand the relation between RF EMF exposure and health and where relevant, to provide a system to protect the

The authors declare no conflicts of interest.

For correspondence contact Gunde Ziegelberger, ICNIRP c/o Bfs, Ingolstaedter Landstr. 1, 85764 Oberschleissheim, or email at Germany. info@icnirp.org.

(Manuscript accepted 14 May 2019)

0017-9078/20/0

Copyright $(\mathrm{C} 2019$ Health Physics Society

DOI: 10.1097/HP.0000000000001137 community from associated harm. Accordingly, the International Commission on Non-Ionizing Radiation Protection (ICNIRP) regularly evaluates the scientific literature and publishes guidelines to restrict non-ionizing radiation in order to protect health. The current guidelines for exposure to RF EMFs were published by ICNIRP two decades ago (ICNIRP 1998), and updated guidelines will soon be published.

An important issue addressed in the scientific literature is whether RF EMFs are carcinogenic. Because there is currently no verified mechanism that would predict that $R F$ EMFs would be carcinogenic (ICNIRP 2009), this issue has primarily been addressed empirically using in vitro, in vivo, or epidemiological methods. In general, in vitro research has tested for effects of RF EMF exposure on a large range of cellular processes that could potentially mediate carcinogenesis, experimental animal-model studies have tested whether cancer rates themselves are affected by RF EMF exposure, and epidemiological research has tested for associations between cancer outcomes and reported usage patterns of devices that utilize RF EMF. As described in the reviews by the Scientific Committee on Emerging and Newly Identified Health Risks (SCENIHR 2015), the Health Council of the Netherlands (HCN 2016), and the Swedish Radiation Safety Authority (SSM 2018), there is currently no evidence and no consensus that RF EMFs are carcinogenic.

However, in terms of experimental animal-model studies, this conclusion is based on the compilation of many individual studies, often employing very different methods of highly discrepant quality. Against this background, the US National Toxicology Program (NTP) initiated a research project to test for effects of GSM (Global System for Mobile communications) and CDMA-modulated (Code-Division Multiple Access) RF EMF exposure over (nearly) the entire lives of a large number of mice and rats, with a particular focus on carcinogenicity (NTP 2018a and b). Contemporaneously, the Ramazzini Institute in Italy conducted an RF EMF base-station exposure carcinogenicity study in rats (Falcioni et al. 2018), which provided a limited set of results directly relevant to the outcomes in the NTP draft reports (NTP 2018c; Wyde et al. 2016). Importantly, unlike the SCENIHR 
(2015), HCN (2016), and SSM (2018) conclusions described above, both studies claimed to have shown that RF EMF exposure is carcinogenic in rats. Should these claims be justified, this would have important ramifications for our current understanding of the impact of RF EMF exposure on health.

Accordingly, ICNIRP has critically evaluated the final NTP reports (2018a and b) and Falcioni et al. (2018) to determine whether they do show that RF EMF exposure is carcinogenic.

\section{SYNOPSIS OF METHODOLOGY AND RESULTS}

\section{Methodology}

To provide context to the critical evaluation of the NTP (2018a and b) and Falcioni et al. (2018) claims that RF EMF exposure is carcinogenic, a brief overview of their methods is given. Although this focuses on aspects of the study that are directly related to carcinogenesis, some additional aspects are described where they relate to issues considered in the discussion.

NTP. Reverberation chambers were used to provide approximately uniform whole-body exposure to groups of 90 male and 90 female Hsd:Sprague Dawley (SD) rats, to either $900 \mathrm{MHz}$ GSM- or CDMA-modulated signals, at specific energy absorption rates (SARs) of 0 (sham), 1.5, 3, or $6 \mathrm{~W} \mathrm{~kg}^{-1}$ (NTP 2018a). Groups of 90 male and 90 female B6C3F1/N mice were also whole-body exposed to $1900 \mathrm{MHz}$ GSM- or CDMA-modulated signals, at SARs of 0 (sham), 2.5 , 5, or $10 \mathrm{~W} \mathrm{~kg}^{-1}$ (NTP 2018b). Note that within each of the rat and mouse arms of the study, a single sham control group was used for both GSM and CDMA group comparisons. Rat and mouse exposures were for $7 \mathrm{~d} \mathrm{wk}^{-1}, 9 \mathrm{~h} \mathrm{~d}^{-1}$ and $10 \mathrm{~min} \mathrm{~d}^{-1}$, given in a $10 \mathrm{~min}$ on/10 min off schedule for $18 \mathrm{~h}$ and $20 \mathrm{~min}$. Male and female rat exposures began in utero (gestation day 5) and continued after birth for up to $107 \mathrm{wk}$. Male and female mouse exposures began at 5-6 wk of age and continued for 106 and $108 \mathrm{wk}$, respectively.

Comparisons were conducted to test whether the sham control group differed from each of the RF EMF-exposed groups and whether there was a trend with increasing exposure. These comparisons were made for a number of biologically relevant end points (e.g., pregnancy rates, pup numbers, body weights, pathologies), separately for mice and rats, males and females, GSM- and CDMA-modulated exposures, at $14 \mathrm{wk}$ (for a subset of animals) and at the end of the $2 \mathrm{y}$ study, and for the total number of organ and tissue pathologies, as well as pathologies per litter. Adjustments for multiple comparisons were not employed, $p$ values $<0.05$ were considered statistically significant, and none of these comparisons were defined a priori as primary end points.
Ramazzini. Although less information was provided regarding the animal exposure in this study, Falcioni et al. (2018) stated that far-field exposures were used to simulate GSM mobile phone base station signals that operated continuously $24 \mathrm{~h} \mathrm{~d}^{-1}$, and that to be representative of real GSM base station emissions, field generation was modulated in the Gaussian minimum shift keying (GMSK) mode, in the "call" operating mode and with complete "Time Slot" assignment. Using this exposure protocol, groups of $>200$ male and $>200$ female rats were whole-body exposed to $1,835 \mathrm{MHz}$ GSM-modulated external fields of 0 (sham control), 5, 25, or $50 \mathrm{~V} \mathrm{~m}^{-1}$ (reported by the authors to be equivalent to whole-body average SARs of $0,0.001,0.03$, or $0.1 \mathrm{~W} \mathrm{~kg}^{-1}$, respectively). Rats were continuously exposed for $19 \mathrm{~h} \mathrm{~d}^{-1}, 7 \mathrm{~d} \mathrm{wk}^{-1}$, beginning in utero (at gestation day 12) and continuing until natural death.

Comparisons tested whether brain and heart pathologies in the sham group differed from the corresponding pathologies in each of the RF EMF-exposed groups and whether there was a trend with increasing exposure. Other comparisons were also conducted that were not specified in Falcioni et al. (2018). Adjustments for multiple comparisons were not employed, $p$ values $<0.05$ were considered statistically significant, and none of the compared pathologies were specified a priori as primary end points.

\section{RESULTS}

The NTP (2018a and b) study provided many thousands of statistical comparisons, addressing both cancer and other outcomes. Evaluation of all comparisons is beyond the scope of the present paper. Instead, we evaluated the strongest NTP claims for RF EMF-induced carcinogenicity, based on the assumption that they are derived from the strongest evidence and thus most relevant to public health. Similarly the Ramazzini study conducted many statistical comparisons, but, as Falcioni et al. (2018) is the only report available and as that paper provided only a subset of the Ramazzini results (those thought to add valuable insight to the NTP conclusions), we evaluated the Ramazzini study only in so far as it helped clarify the NTP conclusions.

In determining what constituted the strongest NTP claims of carcinogenicity, the present paper used the formal NTP ranking system. That system categorizes the evidence resulting from NTP research into "clear evidence," "some evidence," or "no evidence" of carcinogenic activity (or where there are major limitations in the study that preclude interpretation, the study is referred to as "inadequate"). As the designators "clear evidence" and "some evidence" represent, from an NTP perspective, the strongest evidence for carcinogenicity, the following evaluation focuses on the results relating to NTP claims of "clear" and "some" evidence of 
carcinogenicity (NTP 2018a and b). None of the female rat, or the male or female mouse results, were categorized as providing either "clear" or "some" evidence of carcinogenicity and are not discussed further. The NTP claims relating to male rats can be summarized as follows:

1. NTP reported that they found "clear evidence" that RF EMF exposure is carcinogenic due to an increased incidence of malignant schwannoma in the heart of both GSM- and CDMA-exposed rats.

2. NTP reported that they found "some evidence" that RF EMF exposure is carcinogenic due to an increased incidence of malignant glioma in the brain of both GSMand CDMA-exposed rats, and due to an increased incidence of pheochromocytoma (benign, malignant, or complex combined) in the adrenal medulla of GSMexposed rats.

In support of claim 1, NTP reported a statistically significant $(p=0.030)$ increase in malignant schwannoma of the heart in the male rats exposed to CDMA signals at $6 \mathrm{~W} \mathrm{~kg}^{-1}$ (the highest exposure level) and a statistically significant trend in malignant schwannoma incidence as a function of exposure magnitude in both the GSM $(p=0.041)$ and CDMA $(p=0.011)$ conditions (NTP 2018a). In terms of malignant schwannoma, the number of cases in the sham, 1.5, 3, and $6 \mathrm{~W} \mathrm{~kg}^{-1}$ conditions were $0,2,1$, and 5 in the GSM condition, and $0,2,3$, and 6 in the CDMA condition, respectively. Related to this, Falcioni et al. (2018) reported a statistically significant $(p \leq 0.05)$ increase in incidence of heart schwannomas in male rats exposed at $0.1 \mathrm{~W} \mathrm{~kg}^{-1}$ (their highest estimated SAR level), but not at lower exposure levels.

In support of claim 2, NTP reported having found an increased incidence of malignant glioma in the brain of both GSM- and CDMA-exposed male rats. However, the comparisons with the control group were not statistically significant in either the GSM ( $p \geq 0.155)$ or CDMA ( $p \geq 0.221)$ conditions. The number of cases in the sham, $1.5,3$, and $6 \mathrm{~W} \mathrm{~kg}^{-1}$ conditions were $0,3,3$, and 2 in the GSM condition, and 0,0 , 0 , and 3 in the CDMA condition, respectively. The trend in cases over exposure was reported as statistically significant in the CDMA condition only $(p=0.044)$. Within Falcioni et al. (2018), no glioma incidence comparisons were reported to be significant.

In support of claim 2, NTP (2018a) also reported having found an increased incidence of pheochromocytoma (benign, malignant, and complex, combined) in the adrenal medulla of GSM-exposed rats. This was due to a higher incidence in the 1.5 and $3 \mathrm{~W} \mathrm{~kg}^{-1}$ conditions relative to sham $\left(p<0.035, p<0.010\right.$, respectively). The $6 \mathrm{~W} \mathrm{~kg}^{-1}$ condition did not differ from sham $(p=0.472)$, and no trend was observed $(p=0.409)$. Falcioni et al. (2018) did not assess pheochromocytoma lesions.
Due to its potential bearing on the carcinogenicity results, it is important to also consider the rat survival rates. NTP (2018a) reported that survival rates were lower in the male rat control group at the end of the 2-y follow-up period (28\%), compared to the GSM ( $1.5 \mathrm{~W} \mathrm{~kg}^{-1}: 50 \%, p=0.002$; $\left.3 \mathrm{~W} \mathrm{~kg}^{-1}: 56 \%, p \leq 0.001 ; 6 \mathrm{~W} \mathrm{~kg}^{-1}: 68 \%, p \leq 0.001\right)$, and CDMA $\left(1.5 \mathrm{~W} \mathrm{~kg}^{-1}: 48 \%, p=0.005 ; 3 \mathrm{~W} \mathrm{~kg}^{-1}\right.$ : $\left.62 \%, p<0.001 ; 6 \mathrm{~W} \mathrm{~kg}^{-1}: 48 \%, p=0.072\right)$ groups.

\section{DISCUSSION}

There are notable strengths to each of the NTP (2018a and b) and Falcioni et al. (2018) studies. For example, both followed good laboratory practice (GLP), both used much larger numbers of animals than previous research, and both exposed animals over the whole of their lives. This makes it particularly important to consider them in detail given that the conclusions reached in these studies challenge the current scientific consensus in that they each claim to have shown that RF EMF exposure is carcinogenic. If the claims were accurate, and if the research was shown to have relevance to humans, this would represent a crucial issue for ICNIRP to incorporate into the advice and guidance that it provides to the community through a range of formats, such as its RF EMF exposure guidelines.

\section{Context for the NTP and Ramazzini carcinogenicity studies}

Core temperature. When RF EMF is absorbed in tissue, some of the EMF energy is converted to heat. For sufficiently high exposures, the resultant increase in tissue temperature can be harmful. For example, a microwave oven uses very strong RF EMFs to heat food, and, due to the potential for harm associated with inadvertent exposure to these strong EMFs, microwave ovens are engineered so as to preclude human exposure. It follows that there is no need to test whether RF EMF is hazardous, as this is already known. Conversely, the NTP and Ramazzini studies have been designed to test whether particular exposure levels are able to affect health (with exposures ranging from typical human RF EMF exposures in the case of Ramazzini, to RF EMF exposures substantially higher than those that humans are exposed to in the community in the case of NTP). It is important to note though, that as there is no thermal control in either study, neither study is able to comment on whether, if effects were found, they would have been due to thermal or non-thermal mechanisms.

Particularly given that there is currently no evidence of non-thermal mechanisms linking RF EMF effects to health, it is important to understand the RF EMF-induced temperature changes in the NTP and Ramazzini studies. Although the Ramazzini study used exposures that were not high enough to produce appreciable temperature rise, the exposure levels used in NTP would indeed have raised body core 
temperature substantially, which can have important implications for health more generally (ACGIH 2017).

NTP (2018a) measured subcutaneous temperature elevations of approximately $0.7^{\circ} \mathrm{C}$ in the $6 \mathrm{~W} \mathrm{~kg}^{-1}$ exposed rats, during the 1-5 min post-exposure interval, and also reported that the temperature reduced to baseline within 10 min of exposure cessation. A difficulty with this temperature measurement is that the rapid reduction of temperature to baseline makes the delay in measurement result in an underestimate of temperature during exposure, as the temperature would have already been reduced substantially by the time the measurement was made. Further, NTP measured superficial temperature rather than the body core temperature, and superficial temperature will fall faster than body core temperature; body core temperature is unlikely to return to baseline within 10 minutes (Kodera et al. 2017). The magnitude of body core temperature rise in the NTP study is thus difficult to determine, but the indications are that it was substantial. ICNIRP is not aware of research that has tested whether such chronic body core temperature elevations can affect carcinogenicity, but it is striking that there is a preponderance of reported effects in the male rats, whose body core temperature would have increased more than either the female rats or either male or female mice (due to their smaller surface area-to-mass ratio), which would have put them under greater metabolic stress due to their greater thermoregulatory requirements.

\section{Relevance of NTP and Ramazzini exposures to pub-} lic health. Should the claims of NTP (2018a) and Falcioni et al. (2018) be valid, the degree to which their exposure conditions were relevant to public health would be an important consideration. The NTP (2018a and b) studies were not designed to mimic RF EMF levels that people in the community might be exposed to, but rather exposure levels were chosen to be as high as possible without causing gross thermal damage. This is a sensible approach in that if RF EMF was carcinogenic, presumably this would be more clearly seen at extremely high exposure levels. From that point, if carcinogenicity were demonstrated, research could then determine whether similar effects occurred at the lower RF EMF levels that people are exposed to (i.e., whether there is a threshold within the range of current community exposure levels).

The NTP exposures ranged from 1.5 to $6 \mathrm{~W} \mathrm{~kg}^{-1}$ for rats (and from 2.5 to $10 \mathrm{~W} \mathrm{~kg}^{-1}$ for mice), with $6 \mathrm{~W} \mathrm{~kg}^{-1}$ in rats the lowest exposure level reported to elevate malignant cardiac schwannoma incidence. ICNIRP (1998) has both local and whole-body exposure restrictions (basic restrictions). For the general public, local exposure is restricted to $2 \mathrm{~W} \mathrm{~kg}^{-1}$, averaged over any 10 -g mass, and whole-body exposure is restricted to $0.08 \mathrm{~W} \mathrm{~kg}^{-1}$, averaged over the entire body. The NTP exposure of $6 \mathrm{~W} \mathrm{~kg}^{-1}$ is therefore 3 times higher than the local exposure restriction and 75 times higher than the whole-body exposure restriction for the general public. Local and whole-body exposures can produce very different effects, with the latter medically more serious, so the ICNIRP whole-body exposure reduction factors have been set 10 times higher than the equivalent local exposure reduction factors. The NTP (2018a) exposures are thus not directly relevant to those encountered in the community. It follows that, if carcinogenesis was demonstrated, research using substantially lower exposure levels would be required in order to determine whether there was a risk to the public.

The Ramazzini study used whole-body exposures ranging from approximately 0.001 to $0.1 \mathrm{~W} \mathrm{~kg}^{-1}$, which would likely be similar to the local exposures of their rats (given the exposure regime used). This would make all their local exposures within the ICNIRP (1998) exposure restrictions. Their whole-body exposures were also within or similar to the whole-body exposure restrictions from ICNIRP, where their $0.1 \mathrm{~W} \mathrm{~kg}^{-1}$ level only slightly exceeds the ICNIRP whole-body basic restriction of $0.08 \mathrm{~W} \mathrm{~kg}^{-1}$ for the general public. Any positive results could therefore be directly relevant to the ICNIRP (1998) guidelines and the RF EMFs that people in the community may be exposed to.

\section{Basis for NTP's claims that RF EMF exposure is carcinogenic}

As described above, NTP (2018a) based their strongest claims for carcinogenicity on the rat malignant cardiac schwannoma results ("clear evidence"), and on the combination of the rat malignant glioma and pheochromocytoma results ("some evidence").

Malignant cardiac schwannoma. Malignant cardiac schwannomas are extremely rare tumors in humans, and ICNIRP has not found any RF EMF studies that have specifically investigated them in any species. Therefore, this NTP result can only be assessed in terms of internal considerations within the NTP study and for consistency between the NTP (2018a) and Falcioni et al. (2018) studies.

Within the NTP study, effects of RF EMF exposure were reported for male but not female rats, and not for male or female mice. As sex differences are seen elsewhere in carcinogenic processes, the lack of consistency across sexes is plausible, but it reduces the ability to generalize any effects. Similarly, that rats but not mice were reported to be affected would suggest a species-specific effect that reduces the confidence that one can have in generalizing to humans, but does not in itself argue against the veracity of the findings.

However, comparison between the NTP and Ramazzini results is less easy to reconcile. For example, NTP reported an increase in malignant cardiac schwannomas at $6 \mathrm{~W} \mathrm{~kg}^{-1}$ GSM exposure, but not at lower exposures, and reported increases in malignant schwannomas with increasing exposure level in both the GSM and CDMA conditions. Contrary to this, Falcioni et al. (2018) reported an increase at 
approximately $0.1 \mathrm{~W} \mathrm{~kg}^{-1}$, which was not seen in NTP (2018a) exposures that were considerably higher (1.5 and $\left.3 \mathrm{~W} \mathrm{~kg}^{-1}\right)$. It is conceivable that this inconsistency is due to the use of different RF EMF modulation patterns, but there is no evidence in support of this. Further, if this interpretation is correct, it would suggest that the modulation pattern is important to the results, whereas NTP (2018a) argue that similar results are seen in the GSM- and CDMAmodulated signal conditions.

The magnitude of the reported effects is very small. This does not in itself argue against carcinogenicity, but the data are far from robust. This is highlighted in that the control group exhibited lower malignant cardiac schwannomas than has been reported historically (NTP 2018a) and given that an increase of only 1 schwannoma in the control group would have importantly affected the resultant $p$ values (Lee 2016). Particularly given that the same sham control group was used to compare both the GSM- and CDMA-exposure groups, it cannot be determined whether the reported differences would have been due to RF EMF-induced changes, or to abnormalities in the single control group. The statistical strength of the results is also not remarkable. Although NTP (2018a) argue that increased malignant schwannomas of the heart were found in both the GSM and CDMA conditions, it is noteworthy that only the CDMA $6 \mathrm{~W} \mathrm{~kg}^{-1}$ condition was associated with a probability $p<0.05$, and that none of the GSM conditions had $p$ values $<0.05$. Presumably the NTP interpretation was based on the trend analyses, which were associated with $p$ values $<0.05$. However, the trend analyses (as well as the individual comparisons) were complicated by the lower morbidity of the exposed groups (as considered below), making these results difficult to interpret.

Both the NTP (2018a) and Falcioni et al. (2018) studies considered the malignant schwannoma of the heart results to be particularly important because some epidemiological case-control studies, mainly by one research group, reported an increased incidence of vestibular schwannoma from mobile phone use (e.g., Hardell et al. 2005; Hardell and Carlbeg 2009; Hardell et al. 2013). However, neither NTP (2018a and b) nor Falcioni et al. (2018) reported elevated rates of vestibular schwannomas (schwannoma of the auditory nerve, also called acoustic neuroma). NTP (2018a) did find malignant schwannomas in other organs, including the pituitary gland, trigeminal nerve, salivary glands, Harderian gland, eye, thymus gland, uterus, ovary, and vagina. When the incidences of schwannoma in all organs (including the heart) were combined, NTP reported that there was no statistically significant difference from the controls.

Schwann cells are an integral part of all nerves in the peripheral nervous system, and there is no reason to believe that they differ from organ to organ. This also appears to be the position of the NTP (2018a) and Falcioni et al. (2018) study authors because they considered the cardiac schwannoma results to be an important test of the epidemiology reports on increased vestibular schwannoma in higher users of mobile phones. However, testing for evidence in support of the epidemiology vestibular schwannoma reports would require comparing the total number of schwannomas detected at all sites between groups, and not in any individual organ. Alternatively, if the similarity of action of an agent on Schwann cells was disputed, then to test the epidemiology claims, the vestibular schwannoma results would need to be assessed. In each case, assessing the heart separately is not an appropriate comparison for determining whether RF EMF exposure increases the incidence of schwannomas in general or of vestibular schwannomas. Taking a different approach, it might have been reasonable to test for cardiac schwannomas separately if schwannomas in general were of interest, and if exposure was significantly larger in the heart. However, exposure was not larger in the heart in either study, and no argument was made for this approach in either study. Without such hypothesis-driven justification for this particular analysis, the cardiac schwannoma tests can only be considered a subset of the many exploratory comparisons.

Malignant glioma of the brain. Although it is stated that elevated malignant glioma of the brain in both the GSM and CDMA RF EMF groups provides support for the claim that RF EMF exposure is carcinogenic, no differences between sham and exposed groups, in terms of either the GSM or CDMA conditions, differed assuming the $p<0.05$ criterion used in the NTP (2018a) study. The trend over-exposure condition in the GSM groups was also not reported to be statistically significant. Conversely, the trend over-exposure condition in the CDMA groups was reported to be statistically significant $(p=0.044)$, with $0,0,0$, and 3 cases found in the sham, $1.5,3$, and $6 \mathrm{~W} \mathrm{~kg}^{-1}$ groups, respectively. However, as the number of cases did not increase continuously with increasing RF EMF power (the trend is based solely on the $6 \mathrm{~W} \mathrm{~kg}^{-1}$ condition), this does not provide evidence of a graded dose-dependent increase in effect. Further, as the $6 \mathrm{~W} \mathrm{~kg}^{-1}$ condition was not statistically different from the control, it is also difficult to see how this provides support for the supposition that RF EMF CDMAmodulated exposure increases glioma rates. Falcioni et al. (2018) did not find any evidence of increased malignant glioma rates, albeit at lower RF EMF power levels.

Pheochromocytoma of the adrenal medulla. NTP (2018a) claimed that pheochromocytoma (benign, malignant, and complex, combined) of the adrenal medulla also provided evidence that RF EMF exposure was carcinogenic. This was based on increased rates in the 1.5 and $3 \mathrm{~W} \mathrm{~kg}^{-1}$ GSM groups, with no differences seen at the highest exposure condition or in the CDMA conditions. No trends over 
exposure magnitude were reported. ICNIRP is not aware of similar data with which to compare these findings, but if accurate this would suggest that there are differential responses as a function of modulation type and that the effect only occurs within a particular window of exposure magnitude. There are currently no verified mechanisms to explain such complex patterns of effects, and so, as with the malignant cardiac schwannoma and malignant glioma reports, it is important to also consider other explanations for these results.

\section{Methodology and interpretation considerations}

Pathology review procedures. In experimental studies, blinding in the analysis of data is used to ensure that biases related to exposure status and to the determination of outcomes do not affect the outcome of the analysis and thus the results of the study. However, NTP noted that "typically, the initial reading of the slides and the first steps of the pathology review are done by an open, or non-blinded, evaluation by the pathologists involved" (2018a). That is, the initial pathology examination was performed unblinded, and samples where pathology was found (i.e., only a few percent of the total number) were then analyzed by another pathologist who was partially blind to the exposure status (they were told that samples were from "test agent A" or "test agent B" [Wyde et al. 2016]). This does not follow best-practice data analysis procedures and gives substantial potential for biases in the original pathology assessment to affect the study outcomes (e.g., Landis et al. 2012; Bello et al. 2014). Similarly, as noted by a reviewer from the NTP process (Lauer 2016), even identifying samples as being from "test agent A" or "test agent B" can result in bias because perceived patterns within a group's samples can affect how subsequent samples are evaluated.

Whether blinding was used in the Ramazzini study was not specified; however, Falcioni et al. stated that their study was conducted "in compliance with the most recent NTP recommendations" (Falcioni et al. 2018), which suggests that it also has not been conducted blind. Therefore, the degree to which the results in both studies are due to the lack of blinding (as opposed to other factors, such as RF EMF exposure) cannot be determined.

Rat survival rates. An important confounder in the NTP study was that survival was lower and mortality faster in the male rat controls than in the exposed groups $(28 \%$ survival in controls vs. $50-68 \%$ and $48-62 \%$ in the three exposed groups, for GSM and CDMA, respectively). In female rats there was a slightly higher survival in the $6 \mathrm{~W} \mathrm{~kg}^{-1}$ CDMA group (61\%) compared to the control group (41\%). These differences in mortality were reported as statistically significant. Even though the NTP report stated that the better survival of the exposed rats was accounted for statistically, to do so requires mortality and tumor incidence to be independent (in order to model and remove the effect). Instead, the relative contribution from animals that died before the end of the trial was merely reduced (using poly-k weighting). Therefore, there remains a strong possibility that the decrease in survival resulted in underrepresentation of latedeveloping tumors in the controls that importantly affected the statistical results. This reduced mortality pattern was not seen in the Falcioni et al. (2018) study, where all male and female rats, including the controls, survived to term.

Multiple comparisons. A large number of statistical comparisons were conducted in each study without controlling for multiple comparisons; the actual number of analyses is not noted in either study. Although sufficient detail to estimate the number of comparisons has not been provided in Falcioni et al. (2018), from consideration of the NTP $(2018 \mathrm{a}, \mathrm{b})$ reports it can be seen that a range of end points have been assessed (i.e., >200 comparisons), for males and females (i.e., 2), GSM and CDMA (i.e., 2), interim and 2 y intervals (i.e., 2), overall and as a litter ratio separately (i.e., 2), comparing control to each of the three exposure groups as well as the trend over exposure (i.e., 4). This amounts to over 12,800 comparisons, and using a significance level of $p<0.05$, many hundreds are expected to be significant by chance alone; no primary end points were described as a priori hypotheses. It is therefore not possible to determine whether any of the results are due to RF-EMF exposure, as opposed to chance. According to Li et al. (2017), this makes the results useful as "exploratory" analyses only but not as tests of any particular set of hypotheses such as those relating to glioma or malignant cardiac schwannoma. Note that the study also found significant protective effects such as reduced rates of adenoma in exposed female rats compared to the control group, which is also likely to represent a chance finding.

However, it should be noted that the focus of the NTP approach is, for public health reasons, to try to identify potentially harmful agents even if this results in false positives; subsequent research can then test the veracity of the NTP conclusions. Conversely, ICNIRP's role is to determine whether results, such as those from NTP (2018a) and Falcioni et al. (2018), provide evidence that non-ionizing radiation causes harm, and thus it must account for factors such as multiple comparisons.

\section{CONCLUSION}

Although NTP (2018a) and Falcioni et al. (2018) both reported significantly elevated rates of carcinogenic outcomes in male rats, their results are not consistent with each other, nor with the NTP (2018b) mouse or female rat results, nor with the RF cancer literature generally (SCENIHR 2015; HCN 2016; SSM 2018). The NTP's outlying finding is further complicated by important methodological limitations 
including the effect of the greater lifespans of the exposed rats on the statistical analyses, lack of blinding in the pathological analyses, and a failure to account sufficiently for chance in the statistical analyses. Collectively these two studies' limitations preclude drawing conclusions about carcinogenicity in relation to RF EMFs.

Acknowledgments - At the time of draft completion, the ICNIRP Commission was comprised of the following members: Rodney Croft, ${ }^{1}$ Maria Feychting, ${ }^{2}$ Adèle C. Green, ${ }^{3}$ Akimasa Hirata, ${ }^{4}$ Guglielmo d'Inzeo, ${ }^{5}$ Carmela Marino, ${ }^{6}$ Sharon Miller, ${ }^{7}$ Gunnhild Oftedal, ${ }^{8}$ Tsutomu Okuno, ${ }^{9}$ Eric van Rongen, ${ }^{10}$ Martin Röösli, ${ }^{11}$ Zenon Sienkiewicz, ${ }^{12}$ and Soichi Watanabe. ${ }^{13}$

ICNIRP gratefully acknowledges the general support received from the International Radiation Protection Association (IRPA), the German Federal Ministry for the Environment, Nature Conservation, and Nuclear Safety (BMU), the New Zealand Ministry of Health, and the European Union Programme for Employment and Social Innovation (EaSI) (2014-2020). For further information please consult http://ec.europa.eu/social/easi. The information contained in this publication does not necessarily reflect the official position of the European Commission or any other donors. All information concerning the support received by ICNIRP is available at http://www.icnirp.org/en/about-icnirp/support-icnirp/ index.html.

${ }^{1}$ ICNIRP and Australian Centre for Electromagnetic Bioeffects Research, Illawarra Health and Medical Research Institute, University of Wollongong, Australia; ${ }^{2}$ ICNIRP and Karolinska Institute, Sweden; ${ }^{3}$ ICNIRP and QIMR Berghofer Medical Research Institute, Brisbane, Australia, and Australian Centre for International Tropical Health and Nutrition, University of Queensland; ${ }^{4}$ ICNIRP and Nagoya Institute of Technology, Japan; ${ }^{5}$ ICNIRP and La Sapienza University, Rome, Italy; ${ }^{6}$ ICNIRP and Agency for New Technologies, Energy, and Sustainable Economic Development (ENEA), Italy; ${ }^{7}$ ICNIRP and US Food and Drug Administration; ${ }^{8}$ ICNIRP and Norwegian University of Technology (NTNU); ${ }^{9}$ ICNIRP and National Institute of Occupational Safety and Health, Japan; ${ }^{10}$ ICNIRP and Health Council, Netherlands; ${ }^{11}$ ICNIRP and Swiss Tropical and Public Health Institute, Basel, Switzerland; ${ }^{12}$ ICNIRP and Public Health England, United Kingdom; ${ }^{13}$ ICNIRP and National Institute of Information and Communications Technology (NICT), Japan.

\section{REFERENCES}

American Conference of Governmental Industrial Hygienists. ACGIH TLVs and BEIs: based on the documentation of the threshold limit values for chemical substances and physical agents and biological exposure indices. Cincinnati, $\mathrm{OH}$ : ACGIH; 2017.

Bello S, Krogsbøll LT, Gruber J, Zhao ZJ, Fischer D, Hróbjartsson A. Lack of blinding of outcome assessors in animal model experiments implies risk of observer bias. J Clinical Epidemiol 67:973-983; 2014.

Falcioni L, Bua L, Tibaldi E, Lauriola M, De Angelis L, Gnudi F, Mandrioli D, Manservigi M, Manservisi F, Manzoli I, Menghetti I, Montella R, Panzacchi S, Sgargi D, Strollo V, Vornoli A, Belpoggi F. Report of final results regarding brain and heart tumors in Sprague-Dawley rats exposed from prenatal life until natural death to mobile phone radiofrequency field representative of a $1.8 \mathrm{GHz}$ GSM base station environmental emission. Environ Res 165:496-503; 2018.

Hardell L, Carlberg M, Hansson Mild K. Case-control study on cellular and cordless telephones and the risk for acoustic neuroma or meningioma in patients diagnosed 2000-2003. Neuroepidemiol 25:120-128; 2005.

Hardell L, Carlberg M. Mobile phones, cordless phones and the risk for brain tumours. Int J Oncol 35:5-17; 2009.

Hardell L, Carlberg M, Hansson Mild K. Use of mobile phones and cordless phones is associated with increased risk for glioma and acoustic neuroma. Pathophysiol 20:85-110; 2013.

Health Council of the Netherlands. Mobile phones and cancer: part 3. Update and overall conclusions from epidemiological and animal studies. The Hague: Health Council of the Netherlands; Publication 06; 2016.

International Commission on Non-Ionizing Radiation Protection. Guidelines for limiting exposure to time-varying electric, magnetic, and electromagnetic fields (up to $300 \mathrm{GHz}$ ). Health Phys 74:494-522; 1998.

International Commission on Non-Ionizing Radiation Protection. Exposure to high frequency electromagnetic fields, biological effects and health consequences $(100 \mathrm{kHz}-300 \mathrm{GHz})-\mathrm{re}-$ view of the scientific evidence and health consequences. Munich: International Commission on Non-Ionizing Radiation Protection; 2009.

Kodera S, Gomez-Tames J, Hirata A, Masuda H, Arima T, Watanabe S. Multiphysics and thermal response models to improve accuracy of local temperature estimation in rat cortex under microwave exposure. Int J Environ Res Public Health 14(4); 2017. DOI 10.3390/ijerph14040358.

Landis SC, Amara SG, Asadullah K, Austin CP, Blumenstein R, Bradley EW, Crystal RG, Darnell RB, Ferrante RJ, Fillit H, Finkelstein R, Fisher M, Gendelman HE, Golub RM, Goudreau JL, Gross RA, Gubitz AK, Hesterlee SE, Howells DW, Huguenard J, Kelner K, Koroshetz W, Krainc D, Lazic SE, Levine MS, Macleod MR, McCall JM, Moxley RT III, Narasimhan K, Noble LJ, Perrin S, Porter JD, Steward O, Unger E, Utz U, Silberberg SD. A call for transparent reporting to optimize the predictive value of preclinical research. Nature 490:187-191; 2012.

Lauer MS. Reviewer's comments. In: Wyde M, Cesta M, Blystone C, Elmore S, Foster P, Hooth M, Kissling G, Malarkey D, Sills R, Stout M, Walker N, Witt K, Wolfe M, Bucher J. Report of partial findings from the National Toxicology Program carcinogenesis studies of cell phone radiofrequency radiation in Hsd: Sprague Dawley SD rats (whole body exposures). BioRxiv 055699 (preprint 26 May 2016; modified 1 February 2018) [online]. 2016: 50. Available at https:// www.biorxiv.org/content/biorxiv/early/2018/02/01/055699. full.pdf. Accessed 14 May 2019.

Lee MP. Reviewer's comments. In: Wyde M, Cesta M, Blystone C, Elmore S, Foster P, Hooth M, Kissling G, Malarkey D, Sills R, Stout M, Walker N, Witt K, Wolfe M, Bucher J. Report of partial findings from the National Toxicology Program carcinogenesis studies of cell phone radiofrequency radiation in Hsd: Sprague Dawley SD rats (whole body exposures). BioRxiv 055699 (preprint 26 May 2016; modified 1 February 2018) [online]. 2016: 64. Available at https://www. biorxiv.org/content/biorxiv/early/2018/02/01/055699. full.pdf. Accessed 14 May 2019.

Li G, Taljaard M, Van den Heuvel ER, Levine MA, Cook DJ, Wells GA, Devereaux PJ, Thabane L. An introduction to multiplicity issues in clinical trials: the what, why, when and how. Int J Epidemiol 46:746-755; 2017.

National Toxicology Program. Actions from peer review of the draft NTP technical reports on cell phone radiofrequency radiation, March 26-28 [online]. 2018c. Available at https://ntp. niehs.nih.gov/ntp/about_ntp/trpanel/2018/march/ actions20180328_508.pdf. Accessed 14 May 2019.

National Toxicology Program. Toxicology and carcinogenesis studies in Hsd:Sprague Dawley SD rats exposed to wholebody radio frequency radiation at a frequency $(900 \mathrm{MHz})$ and modulations (GSM and CDMA) used by cell phones. Research Triangle Park, NC: National Toxicology Program; NTP TR-595; 2018a.

National Toxicology Program. Toxicology and carcinogenesis studies in B6C3F1/N mice exposed to whole-body radio frequency radiation at a frequency $(1900 \mathrm{MHz})$ and modulations (GSM and 
CDMA) used by cell phones. Research Triangle Park, NC: National Toxicology Program; NTP TR-596; 2018 b.

Scientific Committee on Emerging and Newly Identified Health Risks. SCENIHR opinion on potential health effects of exposure to electromagnetic fields (EMF), 27 January 2015 [online]. 2015. Available at https://ec.europa.eu/health/ sites/health/files/scientific_committees/emerging/docs/ scenihr_o_041.pdf. Accessed 14 May 2019.

Swedish Radiation Safety Authority. Recent research on EMF and health risk - twelfth report from SSM's scientific council on electromagnetic fields [online]. 2018. Available at https://www.stralsakerhetsmyndigheten.se/contentassets/ f34de8333acd4ac2b22a9b072d9b33f9/201809-recentresearch-on-emf-and-health-risk. Accessed 14 May 2019.
Wyde M, Cesta M, Blystone C, Elmore S, Foster P, Hooth M, Kissling G, Malarkey D, Sills R, Stout M, Walker N, Witt K, Wolfe M, Bucher J. Report of partial findings from the National Toxicology Program carcinogenesis studies of cell phone radiofrequency radiation in Hsd: Sprague Dawley SD rats (whole body exposures). BioRxiv 055699 (preprint 26 May 2016; modified 1 February 2018) [online]. 2016. Available at https://www.biorxiv.org/content/biorxiv/early/2018/02/ 01/055699.full.pdf. Accessed 14 May 2019. 